\title{
Comparative Epigenetics Analyses of Acute and Chronic Leukemia
}

\author{
Zhang Yan, Dianjing Guo \\ School of Life Sciences and the State Key Laboratory of Agribiotechnolgy, The Chinese University of Hong Kong, \\ Hong Kong, China \\ Email: djguo@cuhk.edu.hk
}

Received 29 April 2015; accepted 28 June 2015; published 2 July 2015

Copyright (C) 2015 by authors and Scientific Research Publishing Inc.

This work is licensed under the Creative Commons Attribution International License (CC BY).

http://creativecommons.org/licenses/by/4.0/

c) (i) Open Access

\begin{abstract}
Comparative analysis of epigenetic alterations between acute and chronic leukemia, with an emphasis on histone modifications, was conducted. We focused on the promoter regions of the whole genomes as well as oncogenes. Our results revealed that obvious differential histone modifications pattern existed between the two subtypes. H3K27ac had a high tag density in the promoter region in both Dnd41 cell lines and K562 cell lines. H3K27ac and H3K4me1 had high correlation between the two cell lines of oncogenes. Similar results were also achieved in the promoter region of high expression genes in the Jurkat and K562 cell lines based on RNA-seq data. This suggested that $\mathrm{H} 2 \mathrm{~K} 27 \mathrm{ac}$ and $\mathrm{H} 3 \mathrm{~K} 4 \mathrm{me} 1$ were active regulators in leukemia cell lines.
\end{abstract}

\section{Keywords}

Leukemia, Epigenetics, Histone Modification, Promoter

\section{Introduction}

Chronic lymphocytic leukemia (CLL) is the most common leukemia in adults [1] [2]. CLL contains a small fraction of actively proliferating cells, although lymphocytes in the peripheral blood are predominantly resting [3]. Most cases of CLL are preceded by monoclonal B cell lymphocytosis, which is detectable in approximately $5 \%$ of the elderly population and carries a risk of evolving into CLL [4] [5]. Clinically, CLL is characterized by a marked degree of heterogeneity, ranging from patients that harbor highly stable disease with a nearly normal life expectancy to patients with rapidly progressive disease who are destined to succumb in a short time [6] [7].

Acute myeloid leukemia (AML) develops as the consequence of a series of genetic changes in a hematopoietic precursor cell. These changes alter normal hematopoietic growth and differentiation, resulting in an accumulation of large numbers of abnormal, immature myeloid cells in the bone marrow and peripheral blood. 
The exact cause of leukemia is currently unknown although different kinds of leukemia are believed to have different causes, including inherited and environmental (non-inherited) factors. Risk factors for leukemia include previous cancer treatment, genetic disorders, exposure to certain chemicals, smoking and family history of leukemia, etc. [8].

Epigenetic has been defined as the molecular factors and processes around DNA that are mitotically stable and regulate genome activity independent of DNA sequence [8]. Epigenetic research examines biochemical modifications of the genome and aims to determine the resulting consequences on gene function. The two most commonly studied epigenetic mechanisms are DNA methylation and histone modifications [9]. DNA methylation is the most widely studied epigenetic abnormality in tumor genesis. It refers to methylation of cytosine at CpG dinucleotides, and CpG dinucleotides are not randomly distributed throughout the human genome [10]. Loss of DNA methylation at CpG dinucleotides was the first epigenetic abnormality which is to be identified in cancer cells [11]. For instance, hypomethylation leading to activation of genes that are important in cancer includes promoter $\mathrm{CpG}$ demethylation in the overexpression of cyclin D2 and maspin in gastric carcinoma, MN/CA9 overexpression in human renal-cell carcinoma, S100A4 metastasis-associated gene in colon cancer and human papillomavirus 16(HPV16) expression in cervical cancer [11].

Histone tails post-translational modifications are mediated by numerous enzymes, including acetylation, methyla tion, ubiquitination, sumoylation, biotinylation and crotonylation of lysines (K), and phosphorylation of serines (S) and threonines (T) [12]. The pattern of histone modifications, so called "histone code", determines the configuration of chromatin, and adjusts the accessibility to effector proteins [13]. For example, tri-methylation of lysine 4 on histone $\mathrm{H3}$ (H3K4me3) is associated with active transcription, whilst H3K27me3 and H3K9me3 are the two chief repressive marks [14]. There is a large number of enzymes involved in the addition or removal of the covalent modifications, including histone acetyltransferases (HATs), deacetylases (HDACs), methyltransferases (HMTs), and demethylases (HDMs) [14] [15].

Concerning histone modifications, global loss of both acetylation of lysine 16 (H4K16ac) and of trimethylation of lysine 20 of histone H4 (H4K20me3) are commonly found in cancer cells, usually in association with DNA hypomethylation at repetitive DNA sequences [16]. In addition, altered methylation patterns of H3K9 and H3K27 have also been found in cancer cells [17]. Those modifications, which result in inappropriate gene silencing, are accomplished by histone-modifying enzymes. Based on recent study, some researchers found a combinatory effect of different epigenetic modifications on gene expression in breast cancer subtypes, indicating that alterations of epi-modifications in the breast cancer subtypes not only affect genes involved in common cancer biology, but also regulate genes participating in subtype-specific functions [18].

\section{Materials and Methods}

\subsection{Materials}

Epigenetic and transcriptomic data sets of leukemia subtypes are from ENCODE (The Encyclopedia of DNA Elements), GEO (Gene Expression Omnibus) and EMBL-EBI (European Bioinformatics Institute) database (Table 1).

We compare the alterations of five types of histone modifications (H3K4me1, H3K4me3, H3K27ac, H3K36me3 and H3K27me3) and DNA methylation. We use the embryonic stem (ES) cell line and normal blood cell line as control group. The data of ES and normal blood cell lines are both from ENCODE database.

\subsection{Method}

Reads generated from ChIP-Seq and RNA-seq were mapped to human reference genome (hg19) by Bowtie2

Table 1. Description of leukemia subtypes cell lines. AML: Acute myelogenous leukemia, ALL: Acute lym phoblastic leukemia, CML: Chronic myelogenous leukemia.

\begin{tabular}{ccc}
\hline Cell lines & Subtypes of leukemia & Description of cell lines \\
\hline K562 & CML & 53-year-old female CML patient in blast crisis \\
Dnd41 & ALL & 13-year-old boy,with CDKN2B (p15INK4B) and \\
Jurkat & ALL & CDKN2A (p16INK4A) deletions and TP53 (p53) mutation \\
\hline
\end{tabular}


program [19]. To quantify RPKM expression (Reads Per Kilobase of transcript per Million reads mapped) at gene level, the DEGseq program is applied to assemble the reads with default parameters [14]. In this study, we used the middle point of each read as our tag signal. And for each chromatin modification, we used the summary tag counts (STC) obtained at $2 \mathrm{~kb}$ upstream of the transcription start sites (TSSs) as our raw data. The preprocessing steps are described below.

\subsubsection{Calculation of Tag Destiny on the Genome, Promoter and Genes}

For genome wide analysis, promoter regions up to $2 \mathrm{~kb}$ upstream of the transcription start sites (TSSs) were collected from Refseq [20]. Then we use "leukemia subtype" as key word to search the GeneCard database respectively. The analyses of oncogenes promoter can help us to find the real hallmark genes of leukemia subtypes.

ChIP-seq data can be used to locate promoter or CpG Island within few tens of base pairs. Tag density was calculated by STC/2000bp, which means the average tag density of $2 \mathrm{~kb}$ promoter area. Tag densities at the special sites are a good indicator of epigenomic alterations, which makes it easier to quantify and compare epigenomic modifications at different DNA sites.

\subsubsection{Analyses of Differential Histone Modification Sites}

ChIPDiff program [21] was used for genome-wide quantitative comparisons of histone modification. R software package, including rtracklayer, GenomicRanges, Rsamtools and GenomicAlignments was used for statistical analysis.

\section{Results}

\subsection{Differential Histone Modification between CLL and ALL at Chromosomal Level}

Genome wide analysis suggested that there are obvious differential histone modifications in ALL and CLL cell line. The histone modification statistical analyses on the genome chromosome 1 were illustrated in Figure 1.

\subsection{Comparison of Histone Modification in the Promoter Regions}

As shown in Figure 2, Clear difference in histone modification was also observed in the promoter regions between ALL and CLL lines.

Dnd41_chr1_H3K4mel

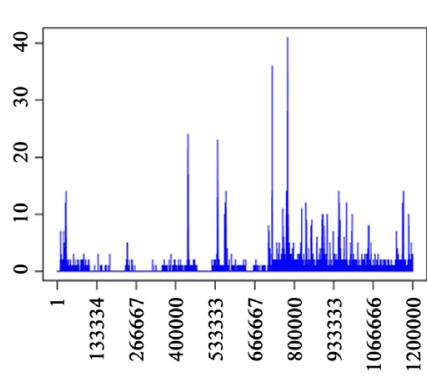

K562_chr1_H3K4me1

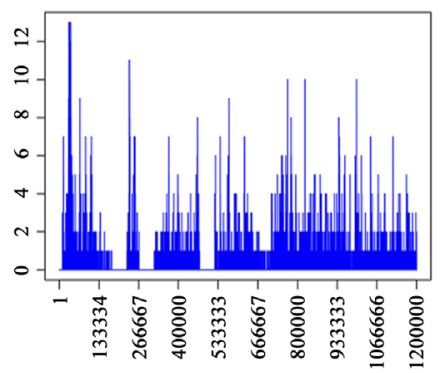

Dnd41_chr1_H3K27ac

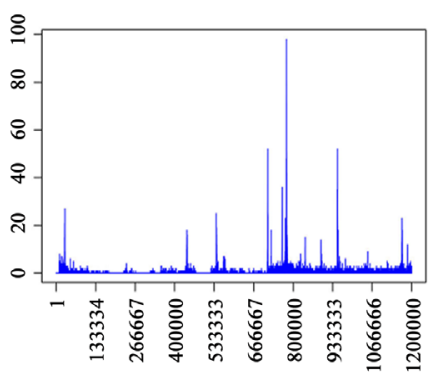

K562_chr1_H3K27ac

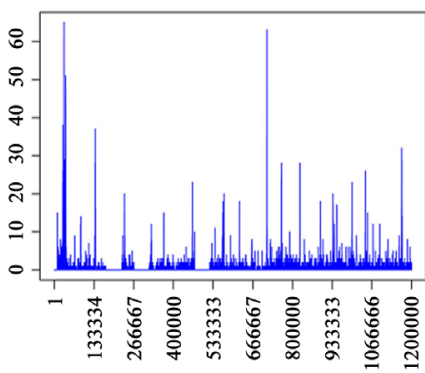

Dnd41_chr1_H3K27ac

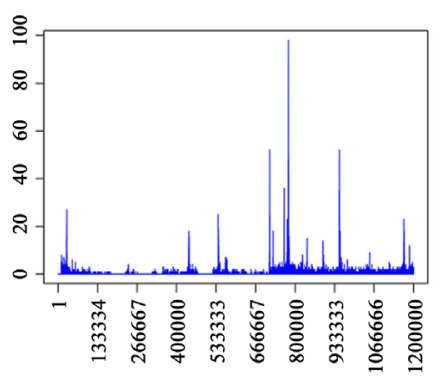

K562_chr1_H3K27me3

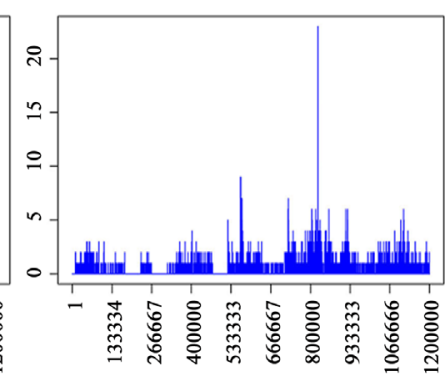

Figure 1. The histone modification patterns for H3K4me1, H3K27ac and H3K27me3 on chromosome 1 of ALL (upper panels) and CLL (lower panels). 

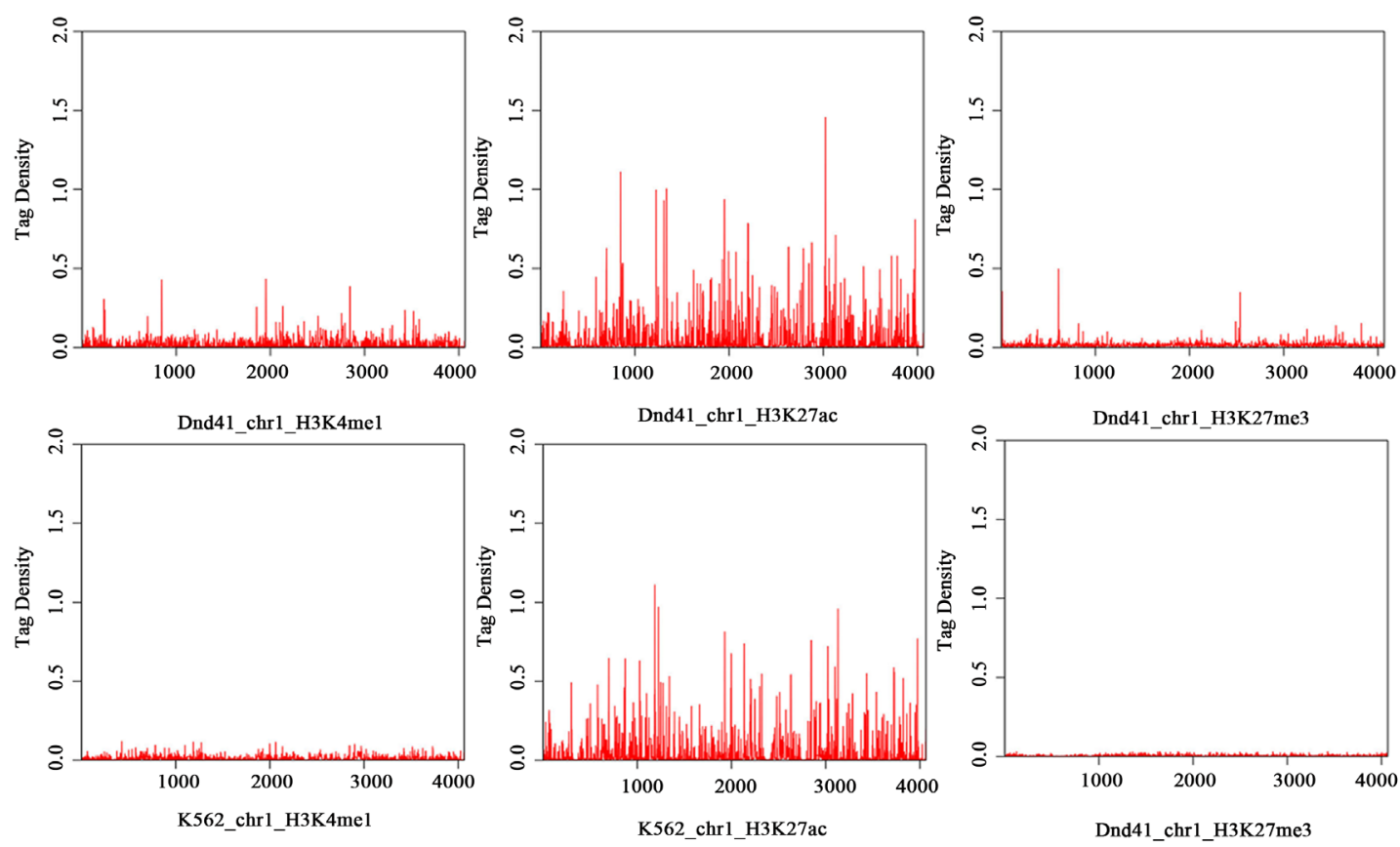

Figure 2. The histone modification pattern for chromosome 1 promoter region of ALL (upper panels) and CLL (lower panels).

\subsection{Density Analysis on the Promoter Regions of Leukemia Oncogenes}

We collected 20 leukemia oncogenes and conducted the density analysis on their promoters. Similiarly, the histone modification patterns are quite different between the two cell lines (Figure 3).

\subsection{Analyses of Gene Expression and Its Correlation with Histone Modifications}

We compared the distribution of the histone modifications on 20 highly expressed gene in ALL and CLL. Of the 4 types of histone modificaions, H3K27ac seems to be the major type (Figure 4).

The correlation of various type of epigenomics modifications and their roles in the regulation of gene expression have drawn lots of attention in research recently. The spearsman correlation matrix of the histone modifications based on the 20 genes suggests that H3K4me1 and H3K27ac have a higher correlation between the two cell lines, and they are both active signals on the promoter area (Figure 5). For the other modifications, e.g. H3K27me3 and H3K36me3 have the low tag density at the promoter region and low correlation between the two cell lines.

We also analyzed the RNA-seq data and calculated the RPKM value with DEGseq software package. Because the RNA-seq data of Dnd41cell were not available, we used the other ALL cell lines Jurkat as the substitute. We first collected 20 high expression genes (RPKM > 100), which appeared in Jurkat cell line and K562 cell line respectively. We then analyzed the distribution of histone modifications (Figure 6).

\section{Discussion}

Epigenetic modifications are recently found to be associated with various cancers. To date, the most thoroughly investigated therapeutic area in terms of epigenetics is cancer [22]. Comparative analyses of epigenetic alterations between the ALL and CLL subtypes of leukemia, especially in histone modifications and DNA methylation, may provide important clue to reveal widespread distinct patterns of epigenetic alterations in leukemia and thereby gain important insight into tumorigenesis.

In this work, we find that H3K27ac has a high tag density in the promoter region in both Dnd41 cell lines and K562 cell lines. H3K27ac and H3K4me1 have high correlation between the two cell lines of oncogenes. Similar results are obtained in the promoter region of high expression genes in the Jurkat and K562 cell lines based on RNA-seq data. This suggests that H2K27ac and H3K4me1 are active regulators in leukemia cell lines. Further 

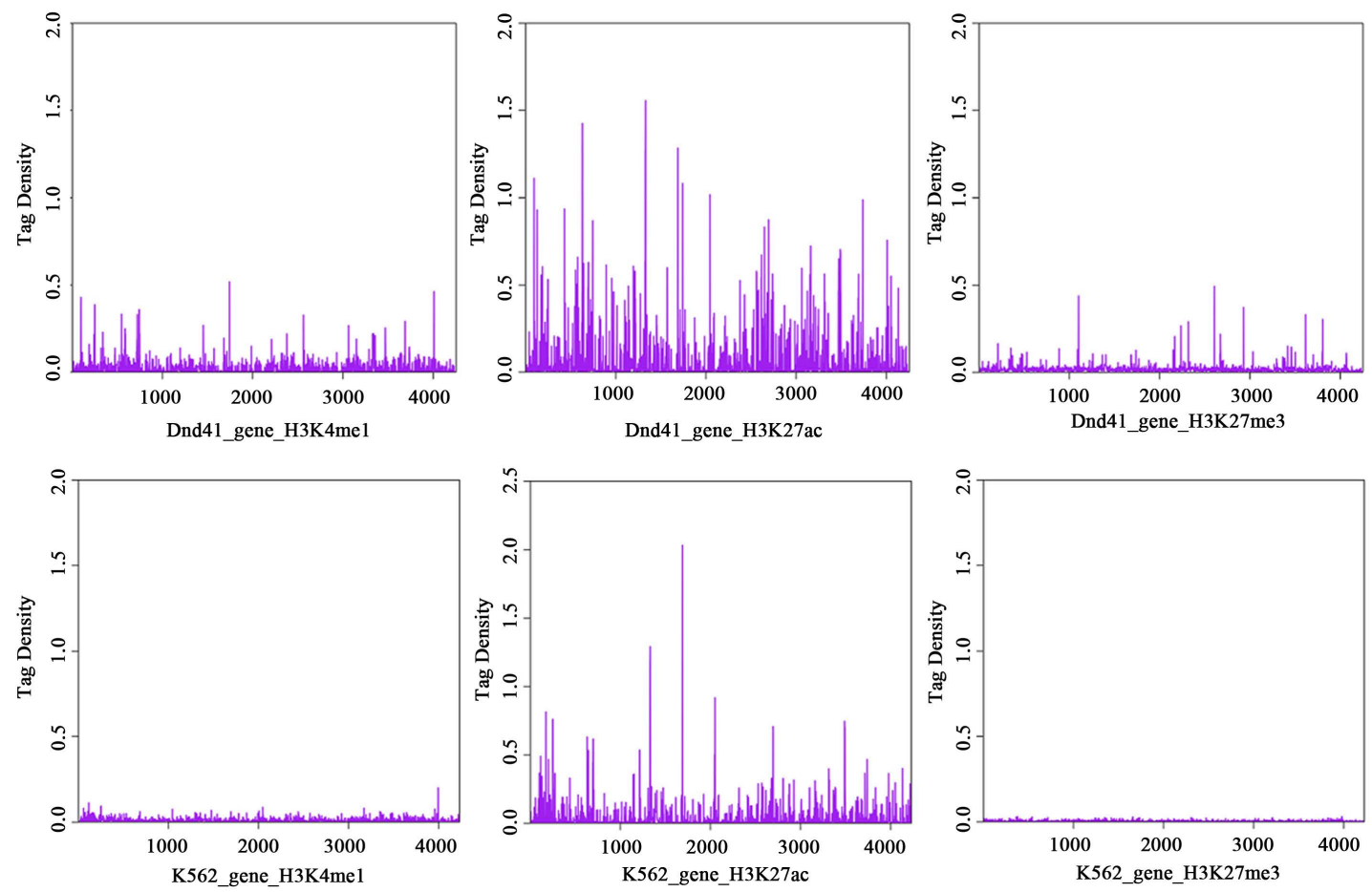

Figure 3. The H3K4me1, H3K27ac and H3K27me3 distribution on promoter of oncogenes in Dnd41 (upper panels) and K562 cell line (lower panels).

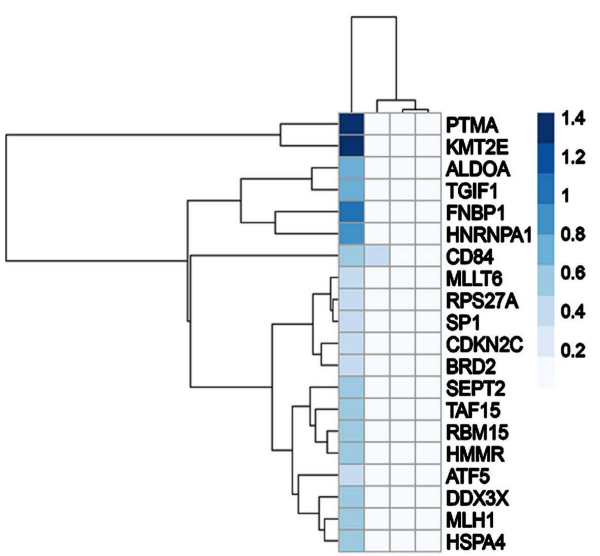

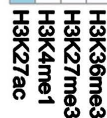

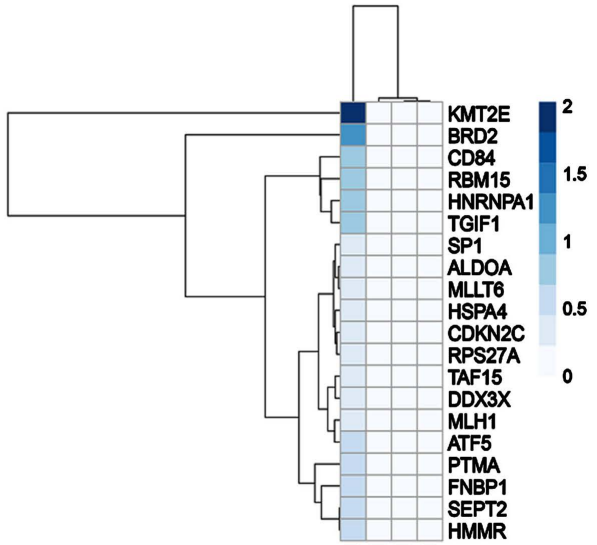

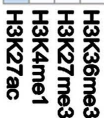

Figure 4. The heat map of 4 types of histone modifications in promoters of 20 highly expressed genes. Left: Dnd41 cell line; Right: K562 cell line.

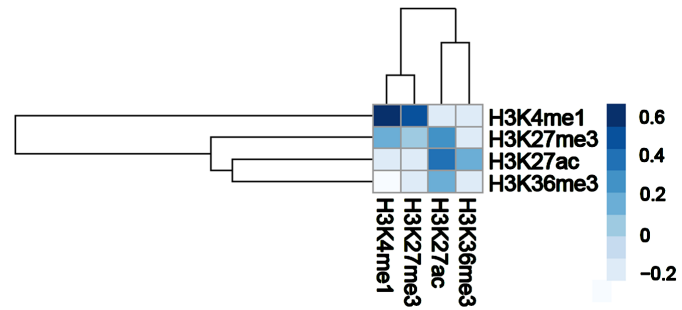

Figure 5. The spearsman correlation matrix of histone modification in Dnd41 and K562 cell lines. 


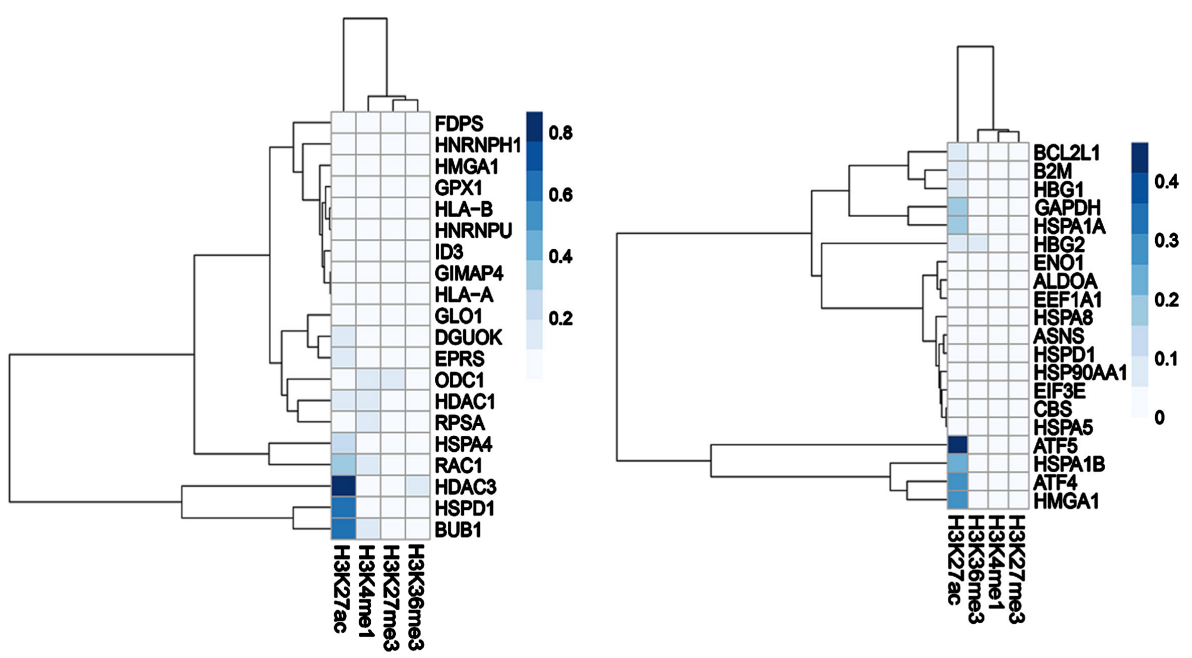

Figure 6. The heat map of 4 types of histone modifications in promoters of 20 highly expressed genes. Left: Jurkat cell line; Right: K562 cell line.

work is being carried out to investigate the correlation between gene expression and epigenetic modification for better understanding the epigenetic effect on leukemia ontogenesis.

Understanding the epigenetic change in AML and CLL may have important therapeutic implications, e.g. targeted therapy aims at correcting the epigenetic defect or its functional consequences. The clinical heterogeneity of CLL often hinders the development of targeted therapy. Translating these molecular prognosticators into the clinic will be critical to improve treatment options in the future.

\section{Acknowledgements}

This work is supported by a grant from the State Key Laboratory of Agribiotechnology at CUHK.

\section{References}

[1] Swerdlow, S.H., Campo, E., Harris, N.L., Jaffe, E.S., Pileri, S.A., Stein, H., et al. (2008) WHO Classification of Tumours of Haematopoietic and Lymphoid Tissues. 4th Edition, World Health Organization, Lyon.

[2] Chiorazzi, N., Rai, K.R. and Ferrarini, M. (2005) Chronic Lymphocytic Leukemia. The New England Journal of Medicine, 352, 804-815. http://dx.doi.org/10.1056/NEJMra041720

[3] Dighiero, G., Travade, P., Chevret, S., Fenaux, P., Chastang, C. and Binet, J.L. (1991) B-Cell Chronic Lymphocytic Leukemia: Present Status and Future Directions. French Cooperative Group on CLL. Blood, 78, 1901-1914.

[4] Rawstron, A.C. (2009) Monoclonal B-Cell Lymphocytosis. ASH Education Book, 2009, 430-439. http://dx.doi.org/10.1182/asheducation-2009.1.430

[5] Rossi, D., Sozzi, E., Puma, A., De Paoli, L., Rasi, S., Spina, V., et al. (2009) The Prognosis of Clinical Monoclonal B Cell Lymphocytosis Differs from Prognosis of Rai 0 Chronic Lymphocytic Leukaemia and Is Recapitulated by Biological Risk Factors. British Journal of Haematology, 146, 64-75. http://dx.doi.org/10.1111/j.1365-2141.2009.07711.x

[6] Shanafelt, T.D., Kay, N.E., Rabe, K.G., Call, T.G., Zent, C.S., Maddocks, K., et al. (2009) Natural History of Individuals with Clinically Recognized Monoclonal B-Cell Lymphocytosis Compared with Patients with Rai 0 Chronic Lymphocytic Leukemia. Journal of Clinical Oncology, 27, 3959-3963. http://dx.doi.org/10.1200/JCO.2008.21.2704

[7] Zenz, T., Gribben, J.G., Hallek, M., Döhner, H., Keating, M.J. and Stilgenbauer, S. (2012) Risk Categories and Refractory CLL in the Era of Chemoimmunotherapy. Blood, 119, 4101-4107. http://dx.doi.org/10.1182/blood-2011-11-312421

[8] Zenz, T., Mertens, D., Küppers, R., Döhner, H. and Stilgenbauer, S. (2010) From Pathogenesis to Treatment of Chronic Lymphocytic Leukaemia. Nature Reviews Cancer, 10, 37-50. http://dx.doi.org/10.1038/nrc2764

[9] Skinner, M.K., Manikkam, M. and Guerrero-Bosagna, C. (2010) Epigenetic Transgenerational Actions of Environmental Factors in Disease Etiology. Trends in Endocrinology \& Metabolism, 21, 214-222. http://dx.doi.org/10.1016/j.tem.2009.12.007

[10] Doherty, R., Farrelly, C.O. and Meade, K.G. (2014) Comparative Epigenetics: Relevance to the Regulation of Produc- 
tion and Health Traits in Cattle. Animal Genetics, 45, 3-14. http://dx.doi.org/10.1111/age.12140

[11] Bhat, A.A., Wani, H.A., Beigh, M.A., Bhat, S.A., Jeelani, S., Massood, A., et al. (2013) Epigenetic Promoter Methylation of Hmlh1 Gene in Human Gut Malignancies: A Comparative Study. Journal of Investigational Biochemistry, 2, 101-108. http://dx.doi.org/10.5455/jib.20130409124009

[12] Feinberg, A.P. and Tycko, B. (2004) The History of Cancer Epigenetics. Nature Reviews Cancer, 4, 143-153. http://dx.doi.org/10.1038/nrc1279

[13] Deakin, J.E., Domaschenz, R., Lim, P.S., Ezaz, T. and Rao, S. (2014) Comparative Epigenomics: An Emerging Field with Breakthrough Potential to Understand Evolution of Epigenetic Regulation. AIMS Genetics, 1, 34-54. http://dx.doi.org/10.3934/genet.2014.1.34

[14] Henrique, R., Luis, A. and Jerónimo, C. (2012) The Epigenetics of Renal Cell Tumors: From Biology to Biomarkers. Frontiers in Genetics, 3, 94. http://dx.doi.org/10.3389/fgene.2012.00094

[15] Shi, Y. (2007) Histone Lysine Demethylases: Emerging Roles in Development, Physiology and Disease. Nature Reviews Genetics, 8, 829-833. http://dx.doi.org/10.1038/nrg2218

[16] Kouzarides, T. (2007) Chromatin Modifications and Their Function. Cell, 128, 693-705. http://dx.doi.org/10.1016/j.cell.2007.02.005

[17] Fraga, M.F., Ballestar, E., Villar-Garea, A., Boix-Chornet, M., Espada, J., Schotta, G., et al. (2005) Loss of Acetylation at Lys16 and Trimethylation at Lys20 of Histone H4 Is a Common Hallmark of Human Cancer. Nature Genetics, 37, 391-400. http://dx.doi.org/10.1038/ng1531

[18] Nguyen, C.T., Weisenberger, D.J., Velicescu, M., Gonzales, F.A., Lin, J.C., Liang, G., et al. (2002) Histone H3-Lysine 9 Methylation Is Associated with Aberrant Gene Silencing in Cancer Cells and Is Rapidly Reversed by 5-Aza-2'deoxycytidine. Cancer Research, 62, 6456-6461.

[19] Langmead, B. and Salzberg, S.L. (2012) Fast Gapped-Read Alignment with Bowtie 2. Nature Methods, 9, $357-359$. http://dx.doi.org/10.1038/nmeth.1923

[20] Wang, L., Feng, Z., Wang, X., Wang, X. and Zhang, X. (2010) DEGseq: An R Package for Identifying Differentially Expressed Genes from RNA-Seq Data. Bioinformatics, 26, 136-138. http://dx.doi.org/10.1093/bioinformatics/btp612

[21] Pruitt, K.D., Tatusova, T. and Maglott, D.R. (2007) NCBI Reference Sequences (RefSeq): A Curated Non-Redundant Sequence Database of Genomes, Transcripts and Proteins. Nucleic Acids Research, 35, D61-D65. http://dx.doi.org/10.1093/nar/gkl842

[22] Xu, H., Wei, C.-L., Lin, F. and Sung, W.-K. (2008) An HMM Approach to Genome-Wide Identification of Differential Histone Modification Sites from ChIP-Seq Data. Bioinformatics, 24, 2344-2349. http://dx.doi.org/10.1093/bioinformatics/btn402 


\section{Appendix}

\section{HATs}

HDACs

HMT

HDMs

ALL

AML

CLL

CML

ENCODE

GEO

EMBL-EBI

ChIP-Seq

RNA-seq

RPKM

Refseq

STC
Histone Acetyltransferases

Histone Deacetylases

Histone Methyltransferases

Histone Demethylases

Acute Lymphoblastic Leukemia

Acute Myelogenous Leukemia

Chronic Lymphocytic Leukemia

Chronic Myelogenous Leukemia

The Encyclopedia of DNA Elements

Gene Expression Omnibus

European Bioinformatics Institute

Chromatin immunoprecipitation (ChIP) followed by high-throughput DNA sequencing

RNA sequencing

Reads Per Kilobase of transcript per Million reads mapped

The Reference Sequence

summary tag counts 